\title{
Brain activations to emotional pictures are differentially associated with valence and arousal ratings
}

\section{Antje B. M. Gerdes ${ }^{1,2+*}$, Matthias J. Wieser ${ }^{1,2+}$, Andreas Mühlberger ${ }^{1}$, Peter Weyers ${ }^{1}$, Georg W. Alpers ${ }^{1,2}$, Michael M. Plichta ${ }^{3}$, Felix Breuer ${ }^{4}$ and Paul Pauli ${ }^{1}$}

\author{
Department of Psychology, University of Würzburg, Würzburg, Germany \\ 2 Chair of Clinical and Biological Psychology, University of Mannheim, Mannheim, Germany \\ ${ }^{3}$ Department of Psychiatry, Central Institute of Mental Health, Mannheim, Germany \\ ${ }_{4}^{4}$ Research Center for Magnetic Resonance Bavaria e.V., Würzburg, Germany
}

Edited by:

Hauke R. Heekeren, Max Planck Institute for Human Development, Germany

\section{Reviewed by:}

K. Luan Phan, University of Michigan, USA

Julia Wendt, Helmut-Schmidt-

University, Germany

*Correspondence:

Antje B. M. Gerdes, University of Mannheim, Chair of Clinical and

Biological Psychology, Ehrenhof Ost,

68131 Mannheim, Germany.

e-mail:gerdes@psychologie.

uni-wuerzburg.de

${ }^{+}$Antje B. M. Gerdes and Matthias J. Wieser are contributed equally to this work
Several studies have investigated the neural responses triggered by emotional pictures, but the specificity of the involved structures such as the amygdala or the ventral striatum is still under debate. Furthermore, only few studies examined the association of stimuli's valence and arousal and the underlying brain responses. Therefore, we investigated brain responses with functional magnetic resonance imaging of 17 healthy participants to pleasant and unpleasant affective pictures and afterwards assessed ratings of valence and arousal. As expected, unpleasant pictures strongly activated the right and left amygdala, the right hippocampus, and the medial occipital lobe, whereas pleasant pictures elicited significant activations in left occipital regions, and in parts of the medial temporal lobe. The direct comparison of unpleasant and pleasant pictures, which were comparable in arousal clearly indicated stronger amygdala activation in response to the unpleasant pictures. Most important, correlational analyses revealed on the one hand that the arousal of unpleasant pictures was significantly associated with activations in the right amygdala and the left caudate body. On the other hand, valence of pleasant pictures was significantly correlated with activations in the right caudate head, extending to the nucleus accumbens (NAcc) and the left dorsolateral prefrontal cortex. These findings support the notion that the amygdala is primarily involved in processing of unpleasant stimuli, particularly to more arousing unpleasant stimuli. Reward-related structures like the caudate and NAcc primarily respond to pleasant stimuli, the stronger the more positive the valence of these stimuli is.

Keywords: emotional pictures, amygdala, caudate, valence, arousal

\section{INTRODUCTION}

During the last decade, several studies were conducted to investigate brain responses to emotional stimuli in healthy subjects (for a summary see Phan et al., 2002; Zald, 2003). Emerging evidence from functional neuroimaging indicates that the amygdala, the ventral striatum including the nucleus accumbens (NAcc), the insula, the anterior cingulate cortex, the medial prefrontal cortex, and the orbitofrontal cortex play the most important role in emotion processing and therefore are considered as key parts of the brain's emotion network (Lang et al., 1998; Garavan et al., 2001; Wright et al., 2001; Hariri et al., 2002; Pessoa and Ungerleider, 2004; Wright et al., 2004). Additionally, it has been repeatedly shown that visual cortex is also more active during emotional compared to neutral visual stimulation (for a review see Lang and Bradley, 2010) most likely due to re-entrant projections from the amygdala (Sabatinelli et al., 2007b). Nevertheless, the specificity of regions like the amygdala to be involved in the processing of pleasant and/or unpleasant information is still under debate (Phan et al., 2004b; Kober et al., 2008). On the one hand, theoretical considerations (LeDoux, 2000; Öhman and Mineka, 2001; Öhman, 2005) as well as experimental findings (Das et al., 2005; Phelps and LeDoux, 2005; Alpers et al., 2009) suggest that the amygdala is mainly involved in the processing of unpleasant and fear-relevant stimuli and responds to the valence of these stimuli (Anders et al., 2008). On the other hand, there is evidence that the amygdala responds to pleasant stimuli as well (Garavan et al., 2001; Baxter and Murray, 2002; Hamann and Mao, 2002; O’Doherty, 2004; Paton et al., 2006; Fecteau et al., 2007; Sabatinelli et al., 2007b; Sabatinelli et al., 2009), which supports the notion of the amygdala as a critical node in relevance detection (Morris et al., 1998; Phan et al., 2002; Zald, 2003; Sergerie et al., 2008). Consequently, the specificity of the amygdala for the processing of unpleasant stimuli is questioned, and it has been suggested that the amygdala is not an exclusive fear module, but tags the emotional salience of high arousing stimuli (Sander et al., 2003). In accordance with this point of view, it has recently been concluded on the basis of $385 \mathrm{fMRI}$ and PET studies, that the amygdala might serve as a general relevance detector of incoming stimuli, whereby unpleasant stimuli may be privileged, but pleasant ones also generate amygdala activation (Costafreda et al., 2008). These findings may be viewed as evidence that arousal drives amygdala activation, rather than valence (Anderson and Sobel, 2003; Small et al., 2003). Presumably, the same holds true for brain areas which are supposed to be mainly involved in processing of pleasant and rewarding stimuli: namely the caudate, the ventral striatum, and the NAcc (McClure et al., 2004; O’Doherty, 2004). Studies employing passive viewing paradigms with pleasant pictures (Aharon et al., 2001; Sabatinelli et al., 2007a), imagery of pleasant scenes (Costa et al., 2010), and reward-processing paradigms (Breiter et al., 2001; 
Knutson and Cooper, 2005; Cooper and Knutson, 2008) indicate that particularly the NAcc is selectively activated by pleasurable material. However, there is also evidence that unpleasant stimuli are processed within these regions (Becerra et al., 2001; Herwig et al., 2007; Carretie et al., 2009; Levita et al., 2009) and a recent review points to NAcc activations in response to both pleasure and pain (Leknes and Tracey, 2007).

The heterogeneous findings regarding the key structures involved in the processing of pleasant and unpleasant pictures may be at least partially the result of the variety of methodological approaches. Most studies investigated unpleasant stimuli only, did not directly compare pleasant and unpleasant stimuli, or incorporated the picture presentation in complex tasks to divert attention (see Phan et al., 2002). Some authors concluded that amygdala activation to unpleasant stimuli mainly occurs under conditions of concurrent attentional load, during indirect tasks or extremely short presentation times (e.g., Pessoa and Ungerleider, 2004; Straube et al., 2008). Furthermore, studies often used only a subset of specific emotion categories (Hariri et al., 2002) or did not match pleasant and unpleasant pictures on the arousal dimension (Lang et al., 1998).

More important, only few studies considered inter-individual differences in emotion processing (Canli et al., 2000; Phan et al., 2003; Anders et al., 2004; Phan et al., 2004a), although inter-individual differences in neural and explicit responses to standardized pictures are presumably large (for a review see Hamann and Canli, 2004). Studies on the association between brain responses and stimulus evaluation revealed that amygdala activity in response to aversive emotional pictures is associated with reported arousal (Phan et al., 2003). In contrast, brain activation related to reward-processing was associated with the individual reward-value (Knutson et al., 2001; Spreckelmeyer et al., 2009), and in response to pleasant pictures, the reward system's activity (e.g., NAcc) seems to be associated with the affective valence of the pictures (Sabatinelli et al., 2007a). To this end, the main goal of our study was to relate subjective judgments of pleasant and unpleasant pictures (valence and arousal ratings) to the neural response to the same pictures to further extend and clarify prior work on individual differences in affective picture processing.

Given the heterogeneous findings on brain areas related either to reward or threat, further research is needed to clarify whether there are certain brain areas which more likely are associated with selfreported valence and arousal (see also Colibazzi et al., 2010). Based on the existing literature on individual differences (see above), one might speculate that activations of specific subsystems in response to aversive stimuli might be more strongly associated with subjective arousal, whereas activations in response to pleasant (rewarding) stimuli might be more associated with subjective pleasantness (reward value).

Thus, we investigated brain responses to pleasant and unpleasant scene pictures with comparable arousal levels and their relationship to ratings of valence and arousal. To enhance statistical power, we presented the stimuli as a block design (see e.g., Friston et al., 1999; Donaldson and Buckner, 2001). Furthermore, it is assumed that blocks of aversive pictures activate motivational circuits related to a general aversive affect, whereas blocks of pleasant pictures induce a pleasant affect. Consequently, the related brain activity reflects the activation of either appetitive or defensive motivational centers (Lang and Bradley, 2010). In addition, we chose to present the pictures in a passive viewing paradigm, as this most reliably elicits amygdala activation (see Costafreda et al., 2008) and possible effects due to task demands are ruled out that way.

Given the findings as reviewed above, we expected to replicate findings of enhanced amygdala responses to unpleasant and enhanced reward system (caudate including the NAcc) responses to pleasant stimuli, and, more interesting, we expected these responses to be associated with subjective levels of valence and arousal.

\section{MATERIALS AND METHODS PARTICIPANTS}

Participants were 17 healthy subjects (eight females) with a mean age of $M=25.12$ years ( $S D=5.02$, range: $20-36)$. Exclusion criteria were pregnancy, psychiatric or neurological problems, psychoactive medication, and left-handedness as assessed by the Edinburgh Handedness Inventory (Oldfield, 1971).

\section{STIMULI, TASK, AND EXPERIMENTAL PROCEDURE}

The stimulus material consisted of 20 pleasant, 20 unpleasant, and 20 neutral pictures taken from the International Affective Picture System (IAPS, Lang et al., 2005) ${ }^{1}$. Based on the normative data, pleasant and unpleasant pictures with similar levels of arousal and an equal distribution of social and non-social pictures were selected.

The experiment was conducted as a block design. Each session consisted of 60 picture stimuli, which were presented in blocks of 10 stimuli. This resulted in six experimental blocks in total, counterbalanced across participants. Two baseline blocks were inserted (cross-hair for $30 \mathrm{~s}$ ). Within each block, the stimuli were displayed for $3 \mathrm{~s}$ without inter-stimulus interval. The duration of the interval between the blocks was $12 \mathrm{~s}$. The stimuli were presented on a light gray background via MRI-compatible goggles (VisuaStim; Magnetic Resonance Technologies, Northridge, CA, USA) using Presentation (Version 9.13, Neurobehavioral Systems, Albany, CA, USA).

The experimental procedure was approved by the ethics committee of the German Psychological Association (DGPs) and written informed consent was obtained. At the beginning of the experimental session, participants were told that their task was to look at the pictures and fixation cross while lying in the scanner. There were three test trials before the experimental part started (15-min duration). After exiting the scanner, participants were asked to rate the perceived valence and arousal (Lang, 1980) of each picture using nine-point Likert-scales (valence 1 = "very unpleasant" to 9 = "very pleasant"; arousal: 1 = "not arousing" to 9 = "highly arousing"). The whole session lasted approximately $50 \mathrm{~min}$.

\section{MAGNETIC RESONANCE IMAGING}

Brain images were acquired using a $1.5 \mathrm{~T}$ whole-body MR tomograph (Magnetom Avanto, Siemens Healthcare, Erlangen, Germany with a quantum gradient system) with a standard 12-channel head

\footnotetext{
${ }^{1}$ IAPS catalog numbers for used pictures: pleasant: 1440, 1999, 2080, 2091, 2216, $4607,4608,4623,4624,4680,5820,5982,7220,7502,7580,8185,8186,8370,8499$, 8502 ; neutral: $1935,2025,2575,2840,2850,5395,5530,5535,6150,7006,7080$, 7170, 7211, 7235, 7247, 7484, 7487, 7491, 8010, 9210; unpleasant: 2352, 2750, 2799, $3230,3300,6200,6250,6300,6415,6550,6821,9007,9253,9265,9300,9342,9561$, 9620, 9830, 9902;
} 
coil and an integrated head holder to reduce head movement. The structural image acquisition consisted of 160 T1-weighted sagittal magnetization-prepared rapid gradient-echo imaging (MP-RAGE) 3D MRI sequence (MPRAGE, 1-mm slice thickness, TR: $2250 \mathrm{~ms}$, TE: $3.93 \mathrm{~ms}$, flip angle: $8^{\circ}$, FOV: $250 \mathrm{~mm}$, matrix: $256 \times 256$, voxel size: $1 \times 1 \times 1 \mathrm{~mm}$ ). For functional imaging, a total of 395 volumes was registered using a $\mathrm{T}_{2}^{*}$-weighted gradient echo-planar imaging sequence (EPI) with 25 axial slices covering the whole brain (5-mm slice thickness; 1-mm gap; interleaved (descending) order; TA: $100 \mathrm{~ms}$; TE: $40 \mathrm{~ms}$; TR: $2.5 \mathrm{~s}$; flip angle: $90^{\circ}$; field of view: $240 \mathrm{~mm} \times 240 \mathrm{~mm}$; matrix size: $64 \times 64$; voxel size: $3.1 \times 3.1 \times 5 \mathrm{~mm}$ ). The orientation of the axial slices was parallel to the AC-PC line.

\section{IMAGE PREPROCESSING AND ANALYSES}

Data were analyzed using Statistical Parametric Mapping (SPM5, Wellcome Department of Cognitive Neurology, London) within MatLab 7.0 (Mathworks Inc., Sherborn, MA, USA). Realignment (b-spline interpolation) was performed (Ashburner and Friston, 2003). To allow localization of functional activation on the subjects' structural MRIs, T1-scans were coregistered to each subject's mean image of the realigned functional images. Coregistered T1 images were then segmented (Ashburner and Friston, 2005), and in the next step, EPI images were spatially normalized into the standard MNI-space using the normalization parameters obtained from the segmentation procedure (voxel size $2 \times 2 \times 2 \mathrm{~mm}^{3}$ ) and spatially smoothed with an 8-mm full-width-half-maximum (FWHM) Gaussian kernel.

The three experimental conditions (unpleasant, pleasant, and neutral) and the fixation blocks were modeled using a boxcar reference vector convolved with a canonical hemodynamic response function (general linear model, Kiebel and Holmes, 2003). The six movement parameters of the rigid body transformation were introduced as covariates. The voxel-based time series were filtered with a high pass filter (cutoff period of $128 \mathrm{~s}$ ). Parameter estimates were subsequently calculated for each voxel (Kiebel and Holmes, 2003) in order to get identical and independently distributed error terms. For each subject, $t$-contrasts were computed: emotional relative to neutral, pleasant relative to neutral, unpleasant relative to neutral, and unpleasant relative to pleasant pictures as well as neutral relative to pleasant and neutral relative to unpleasant, and pleasant relative to unpleasant pictures. For a random effect analysis, the individual contrast images (first-level) were used in a second-level analysis. FMRI data were first analyzed at each voxel (whole brain analysis (WB)) and then specifically for the regions of interest (ROIs). For this analysis, we selected ROIs based on structures which had previously been identified to be relevant for emotional processing (e.g., Lang and Bradley, 2010; Phan et al., 2002): the amygdala ${ }^{2}$, head, and body of caudate including the NAcc, dorsolateral and medial prefrontal cortex, and primary visual areas.

To investigate the brain activity in relation to the subjective ratings, we computed the difference for subjective valence (pleasant - neutral, neutral - unpleasant, pleasant - unpleasant)

\footnotetext{
${ }^{2}$ Amygdala mask was created by combining overlapping voxels of two standard atlases (AAL and Brodmann).
}

and arousal ratings between the emotion categories (pleasant - neutral, unpleasant - neutral, unpleasant - pleasant) and performed a correlation analysis of estimated blood oxygenation level dependent (BOLD) responses of individual first-level contrast images (pleasant relative to neutral, unpleasant relative to neutral, unpleasant relative to pleasant pictures) and the according difference scores.

The WFU Pickatlas software (Version 2.3, Wake Forest University, School of Medicine, NC, USA) was used to conduct the small volume correction with predefined masks in MNIspace (see Tzourio-Mazoyer et al., 2002; Maldjian et al., 2003; Maldjian et al., 2004). For the WB analysis, alpha level was set to $p=0.001$, uncorrected; for the ROI analyses, alpha was set to $p=0.05$ on voxel-level, corrected for multiple comparisons (family-wise error - FWE) and meaningful clusters exceeding five significant voxels.

\section{RESULTS \\ RATINGS}

Analysis showed that the subjective ratings differed as expected between the picture categories, with a main effect of picture category for valence (pleasant: $M=7.18, \mathrm{SD}=0.71$; neutral: $M=5.22$, $\mathrm{SD}=0.37$; unpleasant: $M=2.34, \mathrm{SD}=0.66), F(2,32)=221.76$, $p<0.001$, and arousal (pleasant: $M=5.41, \mathrm{SD}=0.90$; neutral: $M=3.06, \mathrm{SD}=1.06$; unpleasant: $M=5.80, \mathrm{SD}=1.10$ ), $F(2,32)=94.92, p<0.001$.

Follow-up $t$-tests (Bonferroni-corrected) indicated that pleasant pictures were rated as more pleasant than neutral $(t(16)=10.50$, $p<0.001)$ and unpleasant pictures were rated as more unpleasant than neutral ones $(t(16)=16.09, p<0.001)$. Arousal ratings of pleasant and unpleasant pictures did not differ significantly, whereas both categories were rated as more arousing than neutral pictures (pleasant-neutral: $t(16)=16.20, p<0.001$; unpleasantneutral: $t(16)=11.01, p<0.001)$.

\section{HEMODYNAMIC RESPONSES}

\section{Emotional relative to neutral pictures}

The contrast of emotional (pleasant and unpleasant) relative to neutral pictures revealed a significant cluster of increased activation in the left occipital lobe (WB) and the left caudate body (ROI) (see Table 1).

\section{Unpleasant relative to neutral pictures}

The contrast of unpleasant relative to neutral pictures revealed significant activations in left and right occipital regions (WB), the right superior frontal gyrus (WB) and in the left and right amygdala (ROI) (see Table 1).

\section{Pleasant relative to neutral pictures}

The contrast of pleasant relative to neutral pictures revealed significant activations in the left middle temporal gyrus (WB) and left occipital regions (ROI) (see Table 1).

\section{Unpleasant relative to pleasant pictures}

Compared to pleasant pictures, unpleasant pictures strongly activated the left rolandic operculum (WB), the left amygdala (ROI), and the right amygdala (ROI) (see Table 1 and Figure $\mathbf{1}$ ). 
Table 1 | Significant activations revealed by whole brain (WB) and regions of interest (ROI) analysis.

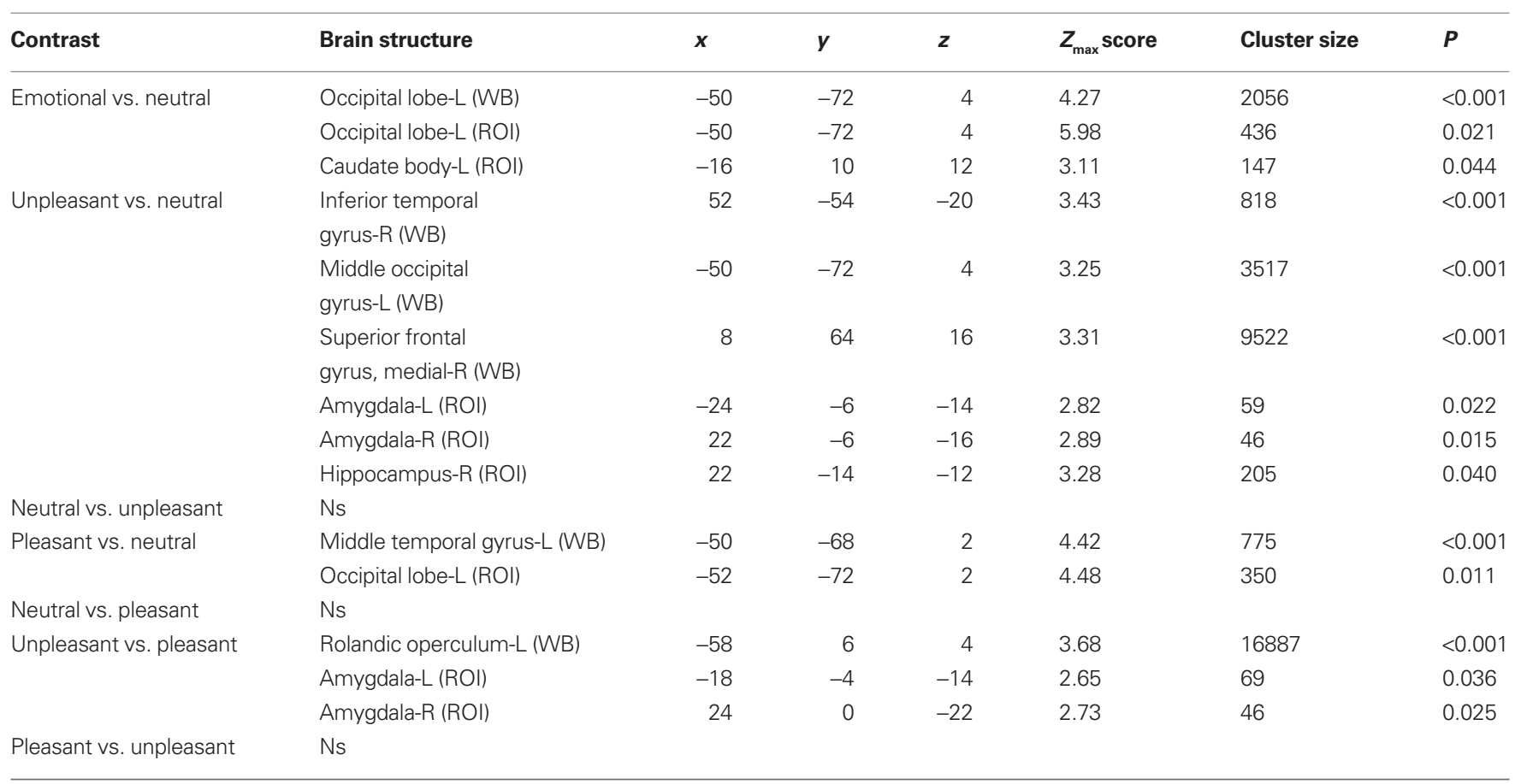

$\alpha<0.001$ uncorrected for whole brain analysis (WB) and $\alpha<0.05$, corrected for multiple comparisons (family-wise error - FWE) in ROI analyses with a minimum cluster size of $k=5$. L, left; $R$, right hemisphere; $n s$, no significant activation. The cluster with the largest number of significant voxels within each region is reported. Coordinates $x, y$, and $z$ of the peak voxels are given in Montreal Neurological Institute space.

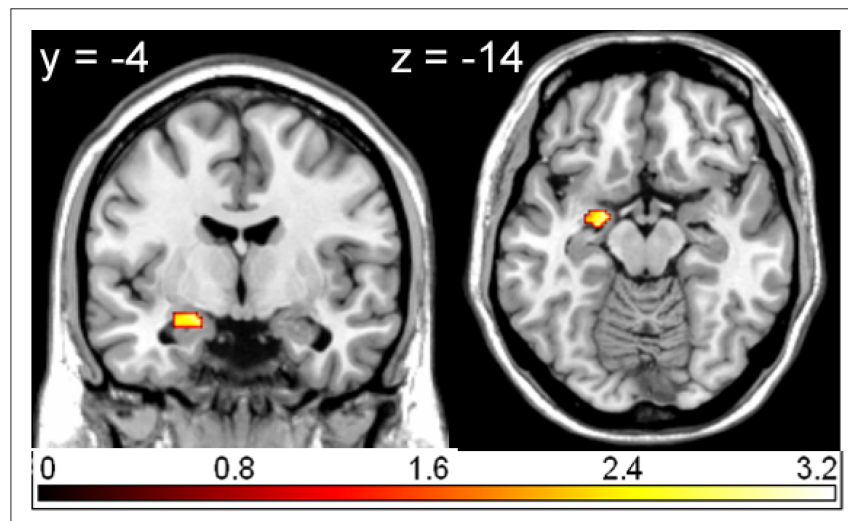

FIGURE 1 | Region of interest (ROI) activation. Left amygdala activation (peak voxel at $-18,-4,-14$ ) for the contrast unpleasant relative to pleasant pictures overlaid on a single subjectT1 scan (overlay threshold: $p<0.05$; minimum cluster size of $k=5$ ). Color-coded t-values are shown.

\section{CORRELATIONAL ANALYSES}

\section{Correlation between valence ratings and brain activations}

In left middle frontal gyrus, the right middle temporal gyrus, the right cerebellum and the superior frontal gyrus (WB), as well as in the head of the right caudate ${ }^{3}$ (ROI), and the left dorsolateral prefrontal cortex (ROI), brain activations in response to pleasant relative to neutral pictures were significantly correlated with the

${ }^{3}$ Correlation coefficient for caudate peak voxel (MNI: $\left.12,22,6\right): r(15)=0.665$, $p=0.004$; correlation coefficient for the averaged cluster: $r(15)=0.546 ; p=0.023$; correlation coefficient for the whole ROI: $r(15)=0.441, p=0.076$. corresponding difference of valence ratings. In other words, the more pleasant the pictures were rated compared to the neutral pictures, the more activation was found in the above-mentioned regions in response to pleasant relative to neutral pictures (see Table 2 and Figure 2).

There were no significant correlations between brain activations in response to unpleasant relative to neutral and unpleasant relative to pleasant pictures and the corresponding valence ratings.

\section{Correlation between arousal ratings and brain activations}

Activations in response to unpleasant relative to neutral pictures in the right amygdala ${ }^{4}$ and the left caudate body (ROI) were found to be significantly correlated with the corresponding arousal ratings (see Table 3 and Figure 3 ). The more arousing participants rated the unpleasant compared to the neutral pictures, the stronger was the activation in these regions.

There were no significant correlations between brain activation in response to pleasant relative to neutral and unpleasant relative to pleasant pictures and the corresponding arousal ratings.

\section{DISCUSSION}

We investigated the neural correlates of pleasant and unpleasant picture processing during a passive viewing task and their relationship to the individual ratings of valence and arousal. First, our results replicate existing findings that the processing of arousing (pleasant and unpleasant) compared to non-arousing pictures is

${ }^{4}$ Correlation coefficient for amygdala peak voxel (MNI: $\left.30,-2,-18\right): r(15)=0.642$, $p=0.005$; correlation coefficient for the averaged cluster: $r(15)=0.568 ; p=0.017$; correlation coefficient for the whole ROI: $r(15)=0.523$; $p=0.031$. 
Table 2 | Significant correlations with valence ratings revealed by whole brain (WB) and regions of interest (ROI) analysis.

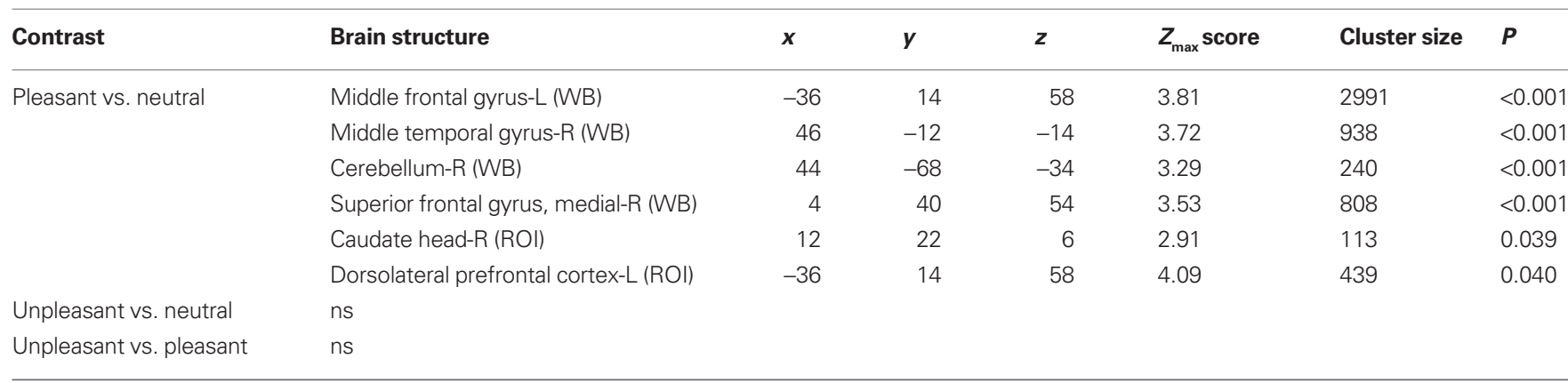

$\alpha<0.001$ uncorrected for whole brain analysis (WB) and $\alpha<0.05$, corrected for multiple comparisons (family-wise error - FWE) in ROI analyses with a minimum cluster size of $k=5 . L=$ left, $R=$ right hemisphere. $n s=$ no significant activation. The cluster with the largest number of significant voxels within each region is reported. Coordinates $x, y$, and $z$ of the peak voxels are given in Montreal Neurological Institute space.

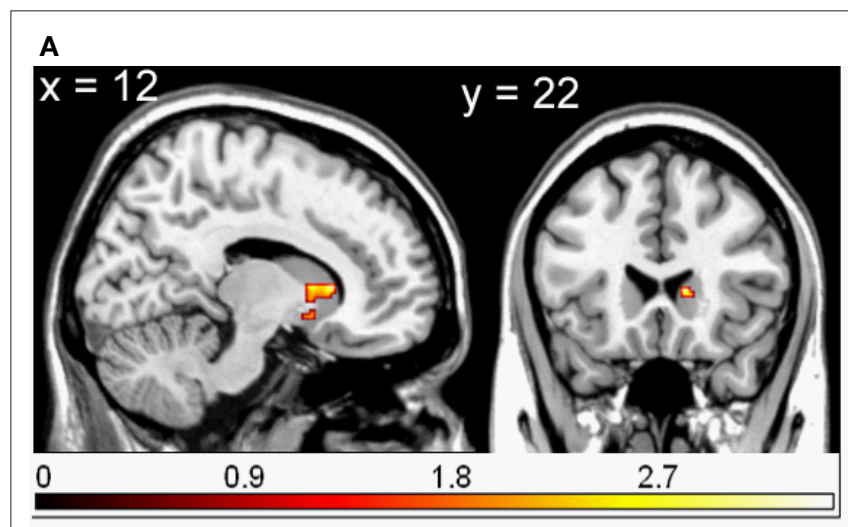

B

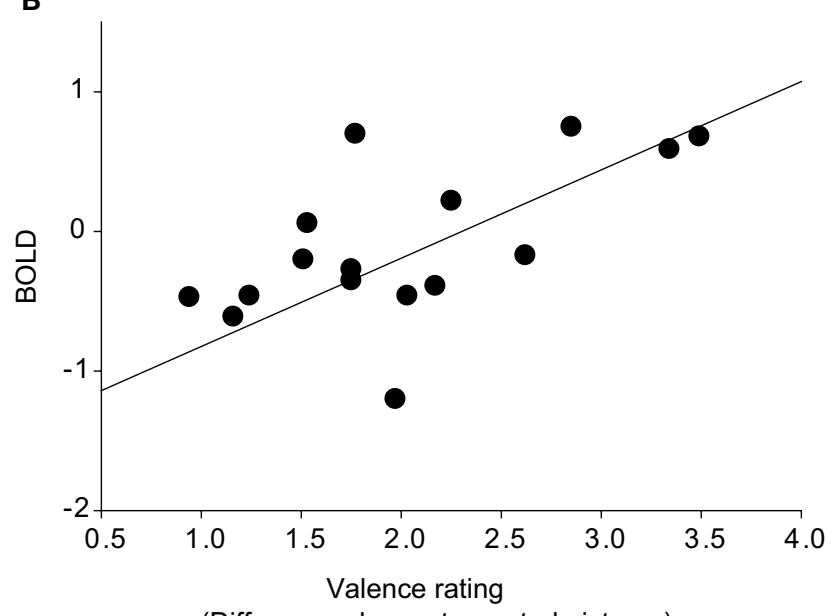

(Difference pleasant - neutral pictures)

FIGURE 2 | Region of interest (ROI) activation. (A) Cluster of significant correlation between right caudate head activation (peak voxel at 12, 22, 6) of the contrast pleasant relative to neutral pictures and subjective valence ratings overlaid on a single subjectT1 scan (overlay threshold: $p<0.05$; minimum cluster size of $k=5$ ). Color-coded t-values are shown. (B) Correlation between right caudate head activation (peak voxel at 12, 22, 6) of the contrast pleasant relative to neutral pictures and subjective valence ratings.

associated with visual cortex activation (Lang et al., 1998). This finding confirms the notion of motivated attention within the visual system for emotionally relevant pictures (Lang and Davis, 2006).
Table 3 | Significant correlations with arousal ratings revealed by whole brain (WB) and regions of interest (ROI) analysis.

\begin{tabular}{llllllll}
\hline Contrast & $\begin{array}{l}\text { Brain } \\
\text { structure }\end{array}$ & $\boldsymbol{x}$ & $\boldsymbol{y}$ & $\boldsymbol{z}$ & $\begin{array}{l}\boldsymbol{Z}_{\max } \\
\text { score }\end{array}$ & $\begin{array}{l}\text { Cluster } \\
\text { size }\end{array}$ & $\boldsymbol{P}$ \\
\hline $\begin{array}{l}\text { Pleasant } \\
\text { vs. neutral }\end{array}$ & $\mathrm{ns}$ & & & & & & \\
$\begin{array}{l}\text { Unpleasant } \\
\text { vs. neutral }\end{array}$ & $\begin{array}{l}\text { Amygdala-R } \\
\text { (ROI) }\end{array}$ & 30 & -2 & -18 & 2.82 & 50 & 0.020 \\
& $\begin{array}{l}\text { Caudate } \\
\text { body-L (ROI) }\end{array}$ & -4 & 20 & -2 & 3.24 & 97 & 0.040 \\
Unpleasant & ns & & & & & & \\
vs. pleasant & & & & & & & \\
\hline
\end{tabular}

$\alpha<0.001$ uncorrected for whole brain analysis (WB) and $\alpha<0.05$, corrected for multiple comparisons (family-wise error - FWE) in ROI analyses with a minimum cluster size of $k=5$. $L$, left; $R$, right hemisphere. ns, no significant activation. The cluster with the largest number of significant voxels within each region is reported. Coordinates $x, y$, and $z$ of the peak voxels are given in Montreal Neurological Institute space.

Second, unpleasant compared to neutral pictures elicited stronger activations in the left and right amygdala, which also stands in line with existing findings (Phan et al., 2002). Third, unpleasant pictures even in comparison to pleasant pictures with comparable normative and actual arousal levels strongly activated the left and the right amygdala. Thus, amygdala activation seems to be related or at least predominantly enhanced in response to unpleasant stimuli.

More importantly, we were able to extend existing findings (Phan et al., 2003, 2004a) by showing that individual ratings can account for individual differences in neural responses to emotional stimuli. For unpleasant pictures, there were strong positive correlations between arousal ratings and right amygdala and left caudate body activation. For pleasant pictures, correlational analysis revealed that valence ratings were associated with activations of the right caudate head including the NAcc, and of the left medial and dorsolateral prefrontal cortex. Thus, correlation of arousal ratings suggests that the amygdala seems to be more involved in the processing of unpleasant pictures, and the strength of this activation is associated with the subjectively experienced arousal level. These findings are in line with existing observations indicating that amgydala responses to unpleasant stimuli 


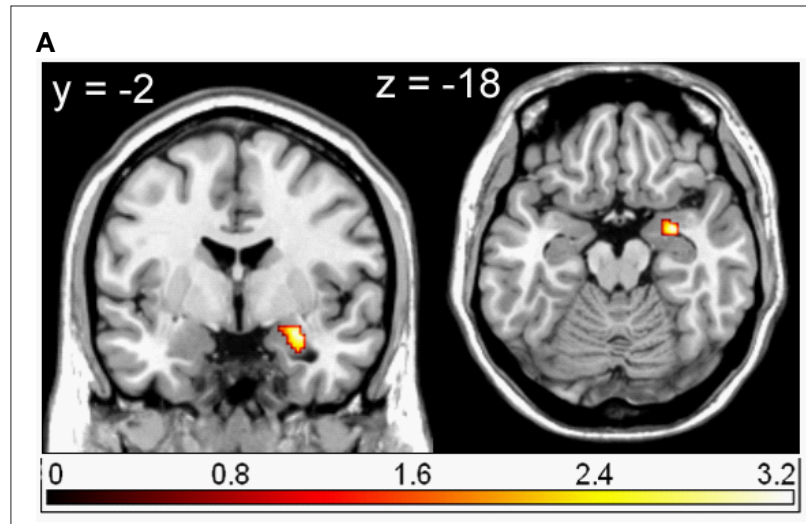

B

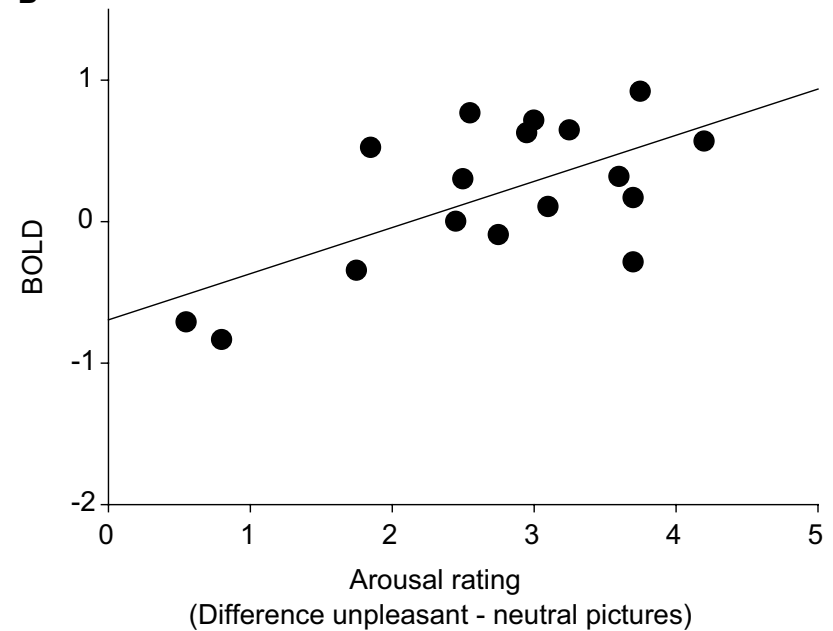

FIGURE 3 | Region of interest (ROI) activation. (A) Cluster of significant correlation between right amygdala activation (peak voxel at $30,-2,-18$ ) of the contrast unpleasant relative to neutral pictures and subjective arousal overlaid on a single subject T1 scan (overlay threshold: $p<0.05$; minimum cluster size of $k=5$ ). Color-coded t-values are shown. (B) Correlation between right amygdala activation (peak voxel at $30,-2,-18$ ) of the contrast unpleasant relative to neutral pictures and subjective arousal.

are dependent on subjective arousal (Canli et al., 2000; Phan et al., 2003) and emphasize the role of this structure in the processing of aversive emotions. Furthermore, the present results nicely corroborate recent findings from Berntson et al. (2007) that patients with an amygdala lesion relative to controls exhibit a complete lack of an arousal gradient across unpleasant stimuli, although they rated the valence of these unpleasant pictures comparable to the control group. Moreover, the patients did not differ from controls regarding responses to pleasant stimuli. These results indicate that the amygdala is important for registering the arousal or the emotional impact specifically of aversive stimuli and is not necessarily involved in the processing of appetitive stimuli (Berntson et al., 2007).

The correlation of caudate body activation and arousal ratings confirms and specifies the involvement of dorsal parts of the caudate in the processing of high arousing unpleasant stimuli
(Carretie et al., 2009) by showing that the subjectively experienced arousal correlates with activation in this area. Similarly, other studies also demonstrated activations of the human dorsal and ventral striatum to non-rewarding cues, including aversive or salient stimuli, and anticipation of negative events (Delgado et al., 2000; Becerra et al., 2001; Jensen et al., 2003; Knutson et al., 2003; Zink et al., 2003).

In contrast, the activation in more ventral parts of the rewardrelated brain areas was found to covary with the valence ratings of pleasant stimuli, but not with arousal ratings, i.e., the more positive subjects rated the pleasant stimuli, the stronger the activations were in the caudate head extending to the NAcc and frontal areas. These observations stand in line with previous findings from neuroimaging studies on reward processing, showing that caudate, NAcc and mPFC are involved in the processing of rewarding (Knutson and Cooper, 2005) and pleasant stimuli (Lane et al., 1997). Furthermore, activations in response to monetary as well as social reward are parametrically modulated by the individual rewarding value of the stimulus (Knutson et al., 2001; Spreckelmeyer et al., 2009). Thus, our findings indicate that the caudate head and the NAcc are involved in the processing of pleasant stimuli and tag their valence rather than the arousal. Thus, one might speculate that different subregions of the caudate are differentially involved in the processing of pleasant and unpleasant pictures (Divac et al., 1967; Yacubian and Buchel, 2009). The absence of significant activation in response to pleasant relative to neutral pictures without consideration of the subjective ratings may indicate that the average rewardvalue of our pleasant pictures is not sufficient enough for all subjects to elicit activation in the reward system, thus subjective differences regarding the reward-values of the cues seem to be very important.

The present findings were realized on the basis of several task characteristics, which might be considered for future studies. First, our results indicate that inter-individual differences in rating of valence and arousal are differentially associated with activities in emotion networks. Therefore, in future neuroimaging studies the additional assessment of subjective ratings seems warranted. Second, our findings occurred when subjects were instructed to simply watch the pictures without any further task, which may be a more ecological valid situation for naturally emerging emotion processing. Consequently, the subjective ratings were assessed after the scanning procedure. Thus, the association between brain activity and the emotional experience may not be as direct as when assessing online rating. Furthermore, a previous study showed that brain activation during passive viewing tasks and brain activation during online ratings can differ (Taylor et al., 2003). However, online and post-scan ratings are shown to be correlated (Phan et al., 2004a) and the cognitive demand due to a rating task may lead to reduced available resources for processing the emotional content.

Third, to obtain an optimal signal-to-noise ratio, a block design without jittered stimulus onset times within the blocks was realized. With this design however, it is not possible to analyze single events, although this might have been interesting with regards to the question whether pleasant stimuli evoke amygdala activation 
or not. In addition, rapid habituation to especially pleasant stimuli may also account for the absence of amygdala activation for the pleasant pictures (Breiter et al., 1996).

In particular from the meta-analysis of Costafreda et al. (2008) it can be assumed that a subset of pleasant stimuli, such as humoristic or erotic material, might evoke amygdala activations to a much higher extend then others. Moreover, in contrast to existing studies (Sabatinelli et al., 2009), we found stronger amygdala activation of unpleasant compared to pleasant pictures. This might be explained by the relatively low arousal level of the emotional pictures used here. Whereas Sabatinelli et al. (2009) used highly arousing affective pictures (erotic, mutilation) for both categories, in our study comparatively low arousing pleasant and unpleasant stimuli were used together with high arousing erotica and mutilations. The conclusion that the amygdala responds already to relatively low arousing aversive stimuli, but probably only to high arousing pleasant stimuli warrants further evaluation.

In sum, our results support the view that amygdala activation predominantly occurs during the processing of unpleasant stimuli, with stronger activations in response to more arousing

\section{REFERENCES}

Aharon, I., Etcoff, N., Ariely, D., Chabris, C. F., O'Connor, E., and Breiter, H. C. (2001). Beautiful faces have variable reward value: fMRI and behavioral evidence. Neuron 32, 537-551.

Alpers, G. W., Gerdes, A. B. M., Lagarie, B., Tabbert, K., Vaitl, D., and Stark, R. (2009). Attention and amygdala activity: an fMRI study with spider pictures in spider phobia. J. Neural Transm. $116,747-757$.

Anders, S., Eippert, F., Weiskopf, N., and Veit, R. (2008). The human amygdala is sensitive to the valence of pictures and sounds irrespective of arousal: an fMRI study. Soc. Cogn. Affect. Neurosci. 3, 233-243.

Anders, S., Lotze, M., Erb, M., Grodd, W., and Birbaumer, N. (2004). Brain activity underlying emotional valence and arousal: a response-related fMRI study. Hum. Brain Mapp. 23, 200-209.

Anderson, A. K., and Sobel, N. (2003). Dissociating intensity from valence as sensory inputs to emotion. Neuron 39, 581-583.

Ashburner, J., and Friston, K. J. (2003). "Rigid body registration," in Human Brain Function, eds S. Zeki, J. T. Ashburner, W. D. Penny, R. S. J. Frackowiak, K. J. Friston, C. D. Frith, R. J. Dolan, and C. J. Price (Oxford: Academic Press), 635-653.

Ashburner, J., and Friston, K. J. (2005). Unified segmentation. Neuroimage 26, 839-851.

Baxter, M. G., and Murray, E. A. (2002). The amygdala and reward. Nat. Rev. Neurosci. 3, 563-573.

Becerra, L., Breiter, H. C., Wise, R., Gonzalez, R. G., and Borsook, D.
(2001). Reward circuitry activation by noxious thermal stimuli. Neuron 32, 927-946.

Berntson, G. G., Bechara, A., Damasio, H., Tranel, D., and Cacioppo, J. T. (2007). Amygdala contribution to selective Affect. Neurosci. 2, 123-129.

Breiter, H. C., Aharon, I., Kahneman, D., Dale, A., and Shizgal, P. (2001). Functional imaging of neural responses to expectancy and experience of monetary gains and losses. Neuron 30, 619-639.

Breiter, H. C., Etcoff, N. L., Whalen, P. J., Kennedy, W. A., Rauch, S. L., Buckner, R. L., Strauss, M. M., Hyman, S. E., and Rosen, B. R. (1996). Response and habituation of the human amygdala during visual processing of facial expression. Neuron 17, 875-887.

Canli, T., Zhao, Z., Brewer, J., Gabrieli, J. D. E., and Cahill, L. (2000). Eventrelated activation in the human amygdala associates with later memory for individual emotional experience. J. Neurosci. 20, 1-5.

Carretie, L., Rios, M., De la Gandara, B. S., Tapia, M., Albert, J., Lopez-Martin, S., and Alvarez-Linera, J. (2009). The striatum beyond reward: caudate responds intensely to unpleasant pictures. Neuroscience 164, 1615-1622.

Colibazzi, T., Posner, J., Wang, Z., Gorman, D., Gerber, A., Yu, S., Zhu, H., Kangarlu, A., Duan, Y., Russell, J.A., and Peterson, B. S. (2010). Neural systems subserving valence and arousal during the experience of induced emotions. Emotion 10, 377-389.

Cooper, J. C., and Knutson, B. (2008). Valence and salience contribute dimensions of emotion. Soc. Cogn.

stimuli. On the contrary, activations in regions discussed to be sensitive to reward were found in response to pleasant stimuli, and these activations increased with the valence of these stimuli, not with their arousal. We conclude that threat-related brain areas like the amygdala show an arousal gradient in their reaction to aversive pictures, whereas activation in reward-related brain areas is linked more to the valence of pleasant pictures. Functionally, the results point to the notion that both amygdala and parts of the caudate including the NAcc are involved in salience detection. However, the processing of threat may more functionally related to arousal, which triggers fight or flight reactions, whereas the response to pleasant stimuli (e.g., food) is more related to the subjective reward-values, which is not necessarily related to high arousal.

\section{ACKNOWLEDGMENTS}

This work was supported by the Research Group "Emotion and Behavior" which is sponsored by the German Research Society (DFG; FOR 605). We would like to thank Christoph Horzella and Jonas Wirbelauer for their help with data collection and data analysis.

to nucleus accumbens activation. Neuroimage 39, 538-547.

Costa, V. D., Lang, P. J., Sabatinelli, D., Versace, F., and Bradley, M. M. (2010). Emotional imagery: assessing pleasure and arousal in the brain's reward circuitry. Hum. Brain Mapp. 31, 1446-1457.

Costafreda, S. G., Brammer, M. J., David, A. S., and Fu, C. H. (2008). Predictors of amygdala activation during the processing of emotional stimuli: a meta-analysis of 385 pet and fMRI studies. Brain Res. Rev. 58, 57-70.

Das, P., Kemp, A. H., Liddell, B. J., Brown, K. J., Olivieri, G., Peduto, A., Gordon, E., and Williams, L. M. (2005). Pathways for fear perception: modulation of amygdala activity by thalamo-cortical systems. Neuroimage 26, 141-148.

Delgado, M. R., Nystrom, L. E., Fissell C., Noll, D. C., and Fiez, J. A. (2000). Tracking the hemodynamic responses to reward and punishment in the striatum. J. Neurophysiol. 84, 3072-3077.

Divac, I., Rosvold, H. E., and Scwarcbart, M. K. (1967). Behavioural effects of selective ablation of the caudate nucleus. J. Comp. Physiol. Psychol. 63, 184-190.

Donaldson, D. I., and Buckner, R. L. (2001). "Effective paradigm design," in Functional Magnetic Resonance Imaging of the Brain: Methods for Neuroscience, eds P. M. Matthews, P. Jezzard, and A. C. Evans (Oxford: Oxford University Press), 177-193.

Fecteau, S., Belin, P., Joanette, Y., and Armony, J. L. (2007). Amygdala responses to nonlinguistic emotional vocalizations. Neuroimage 36, 480-487.

Friston, K. J., Zarahn, E., Josephs, O., Henson, R. N., and Dale, A. M. (1999). Stochastic designs in event-related fMRI. Neuroimage 10, 607-619.

Garavan, H., Pendergrass, J. C., Ross, T. J., Stein, E. A., and Risinger, R. C. (2001). Amygdala response to both positively and negatively valenced stimuli. Neuroreport 12, 2279-2783.

Hamann, S., and Canli, T. (2004). Individual differences in emotion processing. Curr. Opin. Neurobiol. 14, 233-238.

Hamann, S., and Mao, H. (2002). Positive and negative emotional verbal stimuli elicit activity in the left amygdala. Neuroreport 13, 15-19.

Hariri, A. R., Tessitore, A., Mattay, V. S., Fera, F., and Weinberger, D. R. (2002). The amygdala response to emotional stimuli: a comparison of faces and scenes. Neuroimage 17, 317-323.

Herwig, U., Abler, B., Walter, H., and Erk, S. (2007). Expecting unpleasant stimuli-an fMRI study. Psychiatry Res. $154,1-12$.

Jensen, J., McIntosh, A. R., Crawley, A. P., Mikulis, D. J., Remington, G., and Kapur, S. (2003). Direct activation of the ventral striatum in anticipation of aversive stimuli. Neuron 40 , 1251-1257.

Kiebel, S., and Holmes, A. P. (2003). “The general linear model," in Human Brain Function, eds S. Zeki, J. T. Ashburner, W. D. Penny, R. S. J. Frackowiak, K. J. Friston, C. D. Frith, R. J. Dolan, and C. J. Price (Oxford: Academic Press), 725-760. 
Knutson, B., Adams, C. M., Fong, G. W., and Hommer, D. (2001). Anticipation of increasing monetary reward selectively recruits nucleus accumbens. $J$. Neurosci. 21, RC159.

Knutson, B., and Cooper, J. C. (2005). Functional magnetic resonance imaging of reward prediction. Curr. Opin. Neurol. 18, 411-417.

Knutson, B., Fong, G. W., Bennett, S. M., Adams, C. M., and Hommer, D. (2003). A region of mesial prefrontal cortex tracks monetarily rewarding outcomes: characterization with rapid event-related fMRI. Neuroimage 18, 263-272.

Kober, H., Barrett, L. F., Joseph, J., BlissMoreau, E., Lindquist, K., and Wager, T. D. (2008). Functional grouping and cortical-subcortical interactions in emotion: a meta-analysis of neuroimaging studies. Neuroimage 42, 998-1031.

Lane, R. D., Reiman, E. M., Bradley, M. M., Lang, P. J., Ahern, G. L., Davidson, R. J., and Schwartz, G. E. (1997). Neuroanatomical correlates of pleasant and unpleasant emotion. Neuropsychologia 35, 1437-1444.

Lang, P. J. (1980). "Behavioral treatment and bio-behavioral assessment: computer applications" in Technology in Mental Health Care Delivery Systems, eds J. B. Sidowski, J. H. Johnson, and T. A. Williams (Norwood, NJ: Ablex), 119-137.

Lang, P. J., and Bradley, M. M. (2010). Emotion and the motivational brain. Biol. Psychol. 84, 437-450.

Lang, P. J., Bradley, M. M., and Cuthbert, B. N. (2005). International Affective Picture System (IAPS): Affective Ratings of Pictures and Instruction Manual. Technical Report a-6. University of Florida: Gainesville, FL.

Lang, P. J., Bradley, M. M., Fitzsimmons, J. R., Cuthbert, B. N., Scott, J. D., Moulder, B., and Nangia, V. (1998). Emotional arousal and activation of the visual cortex: an fMRI analysis. Psychophysiology 35, 199-210.

Lang, P.J., and Davis, M. (2006). "Emotion, motivation, and the brain: reflex foundations in animal and human research". Progress in Brain Research. 156, 3-29.

LeDoux, J. E. (2000). Emotion circuits in the brain. Annu. Rev. Neurosci. 23, 155-184.

Leknes, S., and Tracey, I. (2007). A common neurobiology for pain and pleasure. Nature 9, 314-320.

Levita, L., Hare, T. A., Voss, H. U., Glover, G., Ballon, D. J., and Casey,
B. J. (2009). The bivalent side of the nucleus accumbens. Neuroimage 44, 1178-1187.

Maldjian, J. A., Laurienti, P. J., and Burdette, J.H. (2004). Precentral gyrus discrepancy in electronic versions of the talairach atlas. Neuroimage 21, 450-455.

Maldjian, J. A., Laurienti, P. J., Kraft, R. A., and Burdette, J. H. (2003). An automated method for neuroanatomic and cytoarchitectonic atlas-based interrogation of fMRI data sets. Neuroimage 19, 1233-1239.

McClure,S.M.,York, M.K., and Montague, P. R. (2004). The neural substrates of reward processing in humans: the modern role of fMRI. Neuroscientist 10, 260-268.

Morris, J.S., Friston, K. J., Buchel, C., Frith, C. D., Young, A. W., Calder, A. J., and Dolan, R. J. (1998). A neuromodulatory role for the human amygdala in processing emotional facial expressions. Brain 121, 47-57.

O’Doherty, J. P. (2004). Reward representations and reward-related learning in the human brain: insights from neuroimaging. Curr. Opin. Neurobiol. 14, 769-776.

Öhman,A.(2005). Therole of the amygdala in human fear: automatic detection of threat. Psychoneuroendocrinology 30 953-958.

Öhman, A., and Mineka, S. (2001). Fears, phobias, and preparedness: toward an evolved of fear and fear learning. Psychol. Rev. 108, 483-522.

Oldfield, R.C. (1971). The assessment and analysis of handedness: the Edinburgh inventory. Neuropsychologia 9 , 97-113.

Paton, J. J., Belova, M. A., Morrison, S. E., and Salzman, C. D. (2006). The primate amygdala represents the positive and negative value of visual stimuli during learning. Nature 439, 865-870.

Pessoa, L., and Ungerleider, L. G. (2004). Neuroimaging studies of attention and the processing of emotion-laden stimuli. Prog. Brain Res. 144, 171-182.

Phan, K. L., Taylor, S. F., Welsh, R. C., Decker, L. R., Noll, D. C., Nichols, T. E., Britton, J. C., and Liberzon, I. (2003). Activation of the medial prefrontal cortex and extended amygdala by individual ratings of emotional arousal: a fMRI study. Biol. Psychiatry 53, 211-215.

Phan, K. L., Taylor, S. F., Welsh, R. C., Ho, S. H., Britton, J. C., and Liberzon, I. (2004a). Neural correlates of individual ratings of emotional salience: a trial- related fMRI study. Neuroimage 21, 768-780.

Phan, K. L., Wager, T. D., Taylor, S. F., and Liberzon, I. (2004b). Functional neuroimaging studies of human emotions. CNS Spectr. 9, 258-266.

Phan, K. L., Wager, T., Taylor, S. F., and Liberzon, I. (2002). Functional neuroanatomy of emotion: a meta-analysis of emotion activation studies in pet and fMRI. Neuroimage 16, 331-348.

Phelps, E. A., and LeDoux, J. E. (2005) Contributions of the amygdala to emotion processing: from animal models to human behavior. Neuron 48, 175-187.

Sabatinelli, D., Bradley, M. M., Lang, P. J. Costa, V. D., and Versace, F. (2007a). Pleasure rather than salience activates human nucleus accumbens and medial prefrontal cortex. J. Neurophysiol. 98 1374-1379.

Sabatinelli, D., Lang, P. J., Keil, A., and Bradley, M. M. (2007b). Emotional perception: correlation of functiona MRI and event-related potentials. Cereb. Cortex 17, 1085-1091.

Sabatinelli, D., Lang, P. J., Bradley, M. M. Costa, V. D., and Keil, A. (2009). The timing of emotional discrimination in human amygdala and ventral visual cortex. J. Neurosci. 29, 14864-14868.

Sander, D., Grafman, J., and Zalla, T. (2003). The human amygdala: an evolved system for relevance detection. Rev. Neurosci. 14, 303-316.

Sergerie, K., Chochol, C., and Armony, J. L. (2008). The role of the amygdala in emotional processing: a quantitative meta-analysis of functional neuroimaging studies Neurosci. Biobehav. Rev. 32, 811-830.

Small, D. M., Gregory, M. D., Mak, Y. E., Gitelman, D., Mesulam, M. M. and Parrish, T. (2003). Dissociation of neural representation of intensity and affective valuation in human gustation. Neuron 39, 701-711.

Spreckelmeyer, K. N., Krach, S., Kohls, G. Rademacher, L., Irmak, A., Konrad, K. Kircher, T., and Grunder, G. (2009). Anticipation of monetary and social reward differently activates mesolimbic brain structures in men and women. Soc. Cogn. Affect. Neurosci. 4 158-165.

Straube, T., Pohlack, S., Mentzel, H. J., and Miltner,W.H. (2008).Differential amygdala activation to negative and positive emotional pictures during an indirect task. Behav. Brain Res. 191, 285-288.

Taylor, S. F., Phan, K. L., Decker, L. R., and Liberzon, I. (2003). Subjective rating of emotionally salient stimuli modu- lates neural activity. Neuroimage 18, 650-659.

Tzourio-Mazoyer, N., Landeau, B. Papathanassiou, D., Crivello, F., Etard, O., Delcroix, N., Mazoyer, B., and Joliot, M. (2002). Automated anatomical labeling of activations in SPM using a macroscopic anatomical parcellation of the MNI MRI single-subject brain. Neuroimage 15, 273-289.

Wright, C. I., Fischer, H., Whalen, P. J., McInerney, S. C., Shin, L. M., and Rauch, S. L. (2001). Differential prefrontal cortex and amygdala habituation to repeatedly presented emotional stimuli. Neuroreport 12 , 379-383.

Wright, P., He, G., Shapira, N. A., Goodman, W. K., and Liu, Y. (2004). Disgust and the insula: fMRI responses to pictures of mutilation and contamination. Neuroreport 15 , 2347-2351.

Yacubian, J., and Buchel, C. (2009). The genetic basis of individual differences in reward processing and the link to addictive behavior and social cognition. Neuroscience 164, 55-71.

Zald, D. H. (2003). The human amygdala and the emotional evaluation of sensory stimuli. Brain Res. Brain Res. Rev. 41, 88-123.

Zink, C. F., Pagnoni, G., Martin, M. E. Dhamala, M., and Berns, G. S. (2003). Human striatal response to salient nonrewarding stimuli. J. Neurosci. 23, 8092-8097.

Conflict of Interest Statement: The authors declare that the research was conducted in the absence of any commercial or financial relationships that could be construed as a potential conflict of interest.

Received: 25 May 2010; paperpending published: 14 June 2010; accepted: 23 August 2010; published online: 28 October 2010. Citation: Gerdes ABM, Wieser MJ, Mühlberger A, Weyers P, Alpers GW, Plichta MM, Breuer F and Pauli P (2010) Brain activations to emotional pictures are differentially associated with valence and arousal ratings. Front. Hum. Neurosci. 4:175. doi:10.3389/fnhum.2010.00175. Copyright (c) 2010 Gerdes, Wieser, Mühlberger, Weyers, Alpers, Plichta, Breuer and Pauli. This is an open-access article subject to an exclusive license agreement between the authors and the Frontiers Research Foundation, which permits unrestricted use, distribution, and reproduction in any medium, provided the original authors and source are credited. 